\title{
Civilisations
}

Revue internationale d'anthropologie et de sciences

humaines

52-2 | 2005

Museums, collections, interpretations

\section{La nature nationale : entre l'universalisme scientifique et la particularité symbolique des nations}

Luiz Fernando Dias Duarte

\section{(2) OpenEdition}

Journals

Édition électronique

URL : http://journals.openedition.org/civilisations/752

DOI : $10.4000 /$ civilisations. 752

ISSN : 2032-0442

\section{Éditeur}

Institut de sociologie de l'Université Libre de Bruxelles

Édition imprimée

Date de publication : 1 juin 2005

Pagination : 21-44

ISSN : 0009-8140

\section{Référence électronique}

Luiz Fernando Dias Duarte, « La nature nationale : entre l'universalisme scientifique et la particularité symbolique des nations », Civilisations [En ligne], 52-2 | 2005, mis en ligne le 02 février 2009, consulté le 19 avril 2019. URL : http://journals.openedition.org/civilisations/752 ; DOI : 10.4000/ civilisations.752 


\title{
La nature nationale: entre l'universalisme scientifique et la particularité symbolique des nations *
}

\author{
Luiz Fernando DIAS DUARTE
}

1. Les musées d'histoire naturelle ont été-parmi les grandes institutions de mémoire

1. inventées dans la culture occidentale moderne -, les principaux responsables d'une tâche difficile : faire accorder le projet de production d'une mémoire, en soi neutre, de l'universalité du savoir scientifique aux exigences de promotion particulière des identités nationales modernes.

Ces deux dimensions fondamentales de l'imaginaire de notre culture ont circonscrit la vocation de ces institutions d'une façon bien plus nette que dans le cas des institutions homologues vouées aux arts et à l'histoire, d'une part, et à la science et la technologie, de l'autre. Dans le premier cas, l'identité nationale s'imposait très clairement; tandis que dans l'autre l'universalité rationnelle trônait sans défi.

Les manières locales d'agencer les combinaisons des deux dimensions ont été très variées dès le $18^{\mathrm{e}}$ siècle; en fonction des caractéristiques et des vicissitudes tant de l'histoire de la raison scientifique que de l'idéal national.

La notion de « nature » a joué un rôle central dans ces agencements symboliques, étant donné sa condition simultanée de référence première de la connaissance scientifique (la " réalité ») et de fonds incontournable des expériences sensibles liées à l'imagerie possible de chaque nation (le « paysage »). La notion même de « culture »-avec ses implications aussi bien imaginaires que scientifiques - ne s'est glissée que très lentement dans cette « nature » primordiale, à travers une série d'opérations de reclassement cosmologique qui se trouvent encore en plein mouvement.

* Version développée d'un exposé au Séminaire ‘Celebrando a Nação'. Museu Histórico Nacional, Rio de Janeiro, 2001. 
On examinera ici les caractéristiques générales de ce processus dans la tradition culturelle occidentale, en choisissant un exemple historique précis : celui du Museu Nacional de Rio de Janeiro, crée en 1818, quatre ans avant l'indépendance et le début de l'implantation de la nation brésilienne ${ }^{1}$.

2 La perspective fondatrice de la culture occidentale moderne est celle de la

2. rationalisation du monde, bien décrite par le concept de désenchantement (Entzauberung) proposé par Max Weber. L'ambition d'un contrôle cognitif et instrumental du monde objectif est un des aspects de ce processus. La « réalité » objective qui succède à la conception d'une unité cosmique subsumée à la divinité (telle la Grande Chaîne des Etres, selon Lovejoy, 1993), acquiert des propriétés d'infinitude spatiale et temporelle (Koyré, 1957) qui permettront la croyance au " progrès de la science » et à la promotion continuelle du bien-être de l'« humanité $»^{2}$.

Ces valeurs suscitent une vigoureuse et continuelle mise en place d'institutions vouées à la science au long du $17^{\mathrm{e}}$ et du $18^{\mathrm{e}}$ siècles. Un vrai marché d'idées scientifiques s'établit, en fonction du souci intrinsèque d'universalisation qui caractérise cette activité. Sociétés savantes, revues scientifiques, instituts de recherche, cabinets de collection, systèmes internationaux d'échange d'informations et d'objets soutiennent et accompagnent le progrès des nouveautés technologiques et la quête de dévoilement des mystères du monde et de dépassement des limites traditionnelles de l'expérience de la nature.

Même si une partie des gouvernements européens s'alignait déjà au $18^{\mathrm{e}}$ siècle à ce qu'on appelle un « despotisme éclairé », c'est-à-dire, à une organisation de l'Etat attentive aux effets de la connaissance scientifique sur la richesse nationale et le bien-être du peuple ${ }^{3}$, ce n'est que sous la Révolution Française et sous Napoléon que s'exprime d'une façon plus évidente et radicale l'association entre les idéaux de la modernité libérale et les vertus du contrôle scientifique de la nature. Peu à peu, on voit se plier à cette injonction même les gouvernements les plus conservateurs. L'esprit révolutionnaire souffle derrière l'ouverture au grand public du Palais du Louvre et du Jardin des Plantes; mais la création du Musée Royal (plus tard National) de Rio de Janeiro, en 1818, sous João VI - qui fuyait justement les troupes napoléoniennes - obéit déjà à des vœux de progrès et bien-être collectif formulés par la propre Couronne.

L'empire de la raison suscitait une importante série de changements dans les régimes de savoir en Occident. On peut souligner d'abord le principe de l'« éloignement du sensible ». A l'avant-garde de la révolution scientifique étaient l'astronomie et la physique, reconstruites selon les projets de Copernic et de Newton. Les modèles

1. Le cas du Brésil peut être particulièrement utile. Les anciennes colonies européennes en Amérique ont dû s'appliquer à produire des identités nationales - au sens moderne - encore plus tôt que la plupart des pays de l'Europe. Elles ont ainsi servi comme des champs d'expérimentation des grands thèmes de l'idéologie nationale du $19^{\mathrm{e}}$ siècle. La considérable stabilité territoriale et politique dont a joui l'Empire brésilien tout au long du siècle rend son cas plus facilement observable.

2. Ce dont fait preuve la fondation à Londres, en 1660, de « The Royal Society of London for Improving Natural Knowledge ».

3. Les effets d'une telle politique ont atteint le Brésil sous le gouvernement du Marquis de Pombal (16991782) - soit directement par les changements dans l'administration coloniale, soit indirectement par les réformes de l'enseignement à l'Université de Coimbra et la création de l'Académie Royale de Sciences de Lisbonne. 
scientifiques proposés dans des domaines aussi différents que la physiologie animale et l'économie politique s'étaient inspirés directement de la perception systématique et abstraite des forces physiques englobantes du poids et du mouvement des masses. Le « mécanisme » avait fourni les images fondatrices de la science occidentale jusqu'à la fin du $18^{\mathrm{e}}$ siècle, lorsque les modèles « organiques » sont devenus acceptables dans le sillage des nouvelles sciences de la « vie » (Gusdorf, 1974).

L'éloignement du sensible intrinsèque aux modèles mécaniques imposa une haute abstraction au concept de « nature », qui a présidé à la conception d'une « nature humaine » universelle et à la proposition d'une connaissance de l'humain également soumise à des règles et modèles scientifiques et universels.

A cette époque, les images de la "liberté » et de l'« égalité » se présentent aussi intensément sous les formules scientifiques que sous les formules politiques. La représentation d'un univers composé de masses discrètes articulées par des règles calculables d'attraction et de répulsion qui se reproduisent sans intervention d'une raison supérieure (pensons au Dieu horloger de Voltaire) est l'homologue du modèle de la pensée humaine organisée comme une association d'idées (Mengal, 2000) et de la nouvelle société humaine comme une association de citoyens libres articulés. Une horreur gnoséologique systématique règne contre toute « différence » a priori, qu'on associe aux superstitions et préjugés censés avoir été abolis par les Lumières (L. Dumont, 1983).

3 Vers la moitié du $18^{\mathrm{e}}$ siècle, on pouvait déjà discerner dans les pays centraux de 3. l'Europe une demande de retour aux liens entre le connaissable et le sensible et une conscience de la spécificité des propriétés de chacun des niveaux de la réalité, sous la forme de « singularités » - c'est à dire, par le moyen d'une curieuse alliance entre le privilège de la partie individuelle et la considération de la «totalité $»^{4}$.

Le principe classificatoire qui range les phénomènes de l'ordre immanent du plus simple au plus complexe fut transposé systématiquement sur le plan historique, diachronique; ce qui entraîna l'ambition d'une corrélation entre l'évolution de la matière et celle de la «civilisation » humaine, renforçant l'impression spontanée de distance entre les sociétés éclairées et les « arriérées » qui se dessinait dès la Renaissance et la découverte de l'Amérique ${ }^{5}$. L'horizon de «perfectibilité » offert à l'espèce humaine inspirait certainement cette vision à la fois dynamique et restrictive, qui servirait de fonds au développement des rapports métropole/colonie typiques du $19^{\mathrm{e}}$ siècle.

On peut aujourd'hui considérer comme la meilleure définition du Romantisme celle de la réaction au désenchantement et à l'éloignement du sensible caractéristiques des Lumières. Son insistance sur la prééminence de la totalité par rapport à la partie, de l'intégration des niveaux de la réalité par rapport à sa distinction heuristique (y comprise surtout celle de l'intellectuel et du sensible), et du flux par rapport à la synchronie, est

4. Louis Dumont (1983 : 210) souligne l'importance du concept de monade chez Leibniz, pour exprimer d'une manière philosophique complexe ce sentiment caractéristique du Romantisme à venir.

5. Ce processus de décalage entre les civilisés et les primitifs ne s'est pas nourri simplement des récits des peuples exotiques. A l'intérieur même de l'Europe, plusieurs conditions culturelles ont servi à la représentation de la primitivité; soit des peuples entiers, tels les Ecossais des Hautes Terres au $18^{\mathrm{e}}$ siècle, selon Lawrence, 1979, ou les Dalmatiens de la même époque, selon Wolff, 2003; soit la paysannerie ou la classe ouvrière urbaine naissante. 
devenue la clé des développements postérieurs des modèles de nature, de nature humaine et de « culture ».

Un des premiers et des plus évidents noyaux de cette inflexion a été la consolidation de l'idée moderne de "nation ». L'imagination de la Renaissance, de la Réforme et des Lumières avait conçu l'idée d'une Humanité, d'un vaste ensemble de tous les êtres humains, indifférenciés - l'homologue, donc, du nouvel univers ouvert et infini. L'insistance romantique sur la singularité est venue distinguer contre ce fonds indifférencié l'unité politique, morale et historique des différentes formations nationales qui se présentaient sur l'échiquier de l'Europe, fournissant donc une nouvelle formule idéologique à l'expérience traditionnelle des différences politiques.

Les collectivités politiques sont venues progressivement à se parer d'attributs d'identité conçus originellement pour les membres individuels des élites européennes à partir de la Renaissance - soit, sa « culture $»^{6}$. Les nations modernes s'instituent ou se réinventent au long du $19^{\mathrm{e}}$ siècle, par un intense travail d'incorporation systématique des trésors accumulés dans son territoire et dans les pratiques de sa population à travers l'histoire. Elles se dotent d'un nouveau sens historique amorcé par ces bizarres amas de références naturelles et fragments culturels. Les philosophes romantiques dressent, à partir de Herder et de Rousseau, la théorie de ces reconstructions, sur l'idée d'un « esprit » (Geist) collectif, redevable d'un culte organisé sur plusieurs niveaux et dans plusieurs temples de la mémoire - dont les nouveaux « musées ». La philologie et la linguistique, les études de folklore, l'archéologie, les «sciences de l'antiquité » et la préoccupation systématique de la préservation d'un passé toujours fuyant surgissent donc au début du $19^{\text {e }}$ siècle, comme autant de jalons sur le chemin de cette nouvelle mémoire nationale.

4 Parallèlement aux voies d'affirmation de ces nouvelles formules du sens de la 4. vie collective, qu'on appelle aujourd'hui « historiques », « sociologiques » ou « anthropologiques », surgit une nouvelle représentation de la propre nature, inspirée du désir de réintégration des abstractions scientifiques dans l'expérience sensible.

L'invention de la géographie moderne est un des signes évidents de cette mouvance, en accord avec l'émergence d'une perspective qu'on peut appeler - d'une façon tout à fait anachronique - d'écologique, au sein même de l'histoire naturelle'. En effet, si le système de classification linnéen avait contribué fort récemment à l'organisation d'une connaissance «naturelle » aussi férue de rationalité et d'universalité que les sciences physiques (Thomas, 1988 : 102) - en dépit de plusieurs décalages imaginaires -, la demande se faisait déjà présente d'une alternative cognitive qui eût pu présenter la connaissance accumulée de la flore et de la faune dans des ensembles diacritiques, où le sens de la « totalité » eût englobé la simple addition des éléments 8 .

6. Il y a un lien historique important, retenu par le lexique, entre la notion traditionnelle de culture agricole (cultiver la nature) et la nouvelle acception, humaine, de «culture » et de "cultivé » (se cultiver). Voir surtout Greenblatt (1980) sur les modèles de la culture de soi pendant la Renaissance et Bruford (1975) sur la tradition de la Bildung romantique.

7. Au Brésil, un mouvement d'intense intérêt pour la conservation de la nature s'est constitué dès la fin du $18^{\mathrm{e}}$ siècle. Il a été bien décrit par J. A. Pádua (2002), surtout à propos de José Bonifácio de Andrada e Silva (1763-1838), le plus important naturaliste et homme d'Etat brésilien du début du $19^{\mathrm{e}}$ siècle.

8. Un pas essentiel de ce processus a été l'œuvre monumentale de Alexandre Von Humboldt : Kosmos (1845) (Ricotta, 2003). 
Un exemple frappant de cette variation holiste, où l'on peut reconnaître aisément l'influence romantique, est celui de la description, proposée par Karl Von Martius dans sa monumentale Flora Brasiliensis, publiée à partir de 1840, des « régions » de la flore brésilienne qu'il avait étudiée in $l o c o^{9}$. Dans le premier volume de cette oeuvre, Von Martius présente un ensemble de Tabulae Physiognomicae, où il décrit les paysages typiques des grandes régions phytogéographiques dans lesquelles il venait d'organiser la connaissance disponible sur l'immense territoire de la récente nation brésilienne. C'est tout à fait révélateur qu'il ait employé la notion de «physiognomonie », dont la version de Lavater était encore récente (M. Dumont, 1984), pour exprimer le rapport entre les dimensions plus impressionnistes, sensibles, des «paysages » naturels ${ }^{10}$ et les essais de compréhension universaliste du monde naturel (1995). La physiognomonie peut être considérée comme une des premières manifestations systématiques du projet d'une science romantique, forte de l'union du sensible et du cognitif, dans le style de la Naturphilosophie. Pour symboliser ces physionomies régionales, Von Martius a choisi dans la mythologie grecque les noms de différents types de nymphes - ce qui n'est pas sans évoquer l'esprit des correspondances selon le goût de la Renaissance.

La catégorie de «vie», centrale pour les nouveaux horizons de la science du $19^{\mathrm{e}}$ siècle, exprime très bien cette combinaison désirée entre l'ambition universaliste et les distinctions romantiques (Gusdorf, 1974). En effet, son émergence impose la reconnaissance de seuils différenciés de la réalité, dans l'opposition, par exemple, entre le monde animé et l'inanimé. Elle exige aussi bien une certaine considération de la totalité et de la singularité - qui est venue s'exprimer dans le concept d' " organisme ». Le concept de « vie » entraîne finalement une primauté du flux temporel, de la transformation, du devenir, du développement vital - dont s'occuperaient les successives théories d'une évolution naturelle. On ne peut pas oublier que le terme «biologie » n'a été proposé qu'en 1802 (Mengal, 2000).

On aurait pu dire autant de la présence de ces éléments épistémologiques dans l'instauration de la science géologique et - puis - de la paléontologie : identique intérêt aux « formations », en tant qu'unités phénoménales (rapprochables de l'idée de Gestalt ou de forme structurelle ${ }^{11}$ ); identique encadrement dans des séries de transformations évolutives. La notion même du temps moderne est censée avoir été instaurée par la démonstration d'un nouveau «temps géologique » - sans commune mesure avec la temporalité occidentale traditionnelle (Gould, 1987).

En réalité, la quête de connaissance et de préservation d'une «nature » douée des attributs qu'on vient de décrire obéissait à la même pulsion des sciences historiques :

9. Karl Friedrich Von Martius (1794-1868) était un naturaliste allemand, invité au Brésil dans le cadre d'une mission scientifique envoyée par l'Empereur d'Autriche (et le Roi de Bavière) à l'occasion du mariage de sa fille Léopoldine - elle-même dévouée à la science naturelle - au Prince Pedro, futur Empereur du Brésil.

10. On ne peut pas oublier que l'apogée du genre des peintures de 'paysage' est contemporain de l'investissement romantique sur le rapport de l'expérience de la nature aux états d'âme individuels et aux « sentiments nationaux » (Thomas, 1988). La catégorie elle-même provient de l'italien paesaggio, où la racine paese (pays) est encore plus évidente. En allemand et en anglais, Landschaft et landscape ont une étymologie équivalente.

11. Les rapports entre l'idée romantique d'une forme originaire, la notion de formation géologique et la notion de structure dans l'œuvre de Claude Lévi-Strauss ont été fort bien développées par Carlo Severi (1988). 
la notion d'une « histoire naturelle » englobait finalement les phénomènes biologiques, géologiques et anthropologiques. La "science des voyageurs », si caractéristique du $19^{\mathrm{e}}$ siècle et si intimement liée à l'épanouissement des musées d'histoire naturelle, est un bon exemple de la combinaison entre l'ambition universaliste de la science et les différents seuils de l'expérience sensible, dont la complexité hante tous les esprits. Les ressources des arts plastiques et de l'expression littéraire sont mises au service de la besogne descriptive et classificatoire, ce qui induit des rapprochements curieux entre la science et l'art officiel de cette période ${ }^{12}$. L'image même du «voyage »d'aventure, physique et intellectuel, évoquait la tradition des voyages de formation des élites européennes et se prêtait aux nouvelles exigences de la Bildung personnelle romantique (qu'on songe aux Rêveries de Rousseau et aux Wandersjahren de Goethe). Entre Chateaubriand au Mississipi, Agassiz en Amazonie, Darwin aux Galápagos, Gauguin à Tahiti, Rimbaud en Ethiopie, un grand pont de transports et déplacements lie la fin du $18^{\mathrm{e}}$ au début du $20^{\mathrm{e}}$ siècle (Nery, 1996).

5 Les images de cette nature surchargée de significations se croisent avec celles

5 . qui viennent consacrer le nouveau statut des « nations ». Parmi les multiples qualités et capacités qu'elles portent, celle du « territoire » est particulièrement intense. C'est justement autour de cette notion que viendront s'établir et s'offrir à une vaste consommation symbolique les « natures nationales » modernes.

La matière est longue, en dépit de sa courte histoire. Peu d'entreprises symboliques auront exigé dans notre culture un investissement aussi intense et systématique. Les rapports entre l' " esprit » national et les caractéristiques de la nature de son « territoire » se sont inspirés probablement au début des anciennes théories humorales, qui proposaient des continuités cosmologiques, "sympathiques ", entre la constitution des peuples et celle des terres qu'ils habitaient (par exemple, les Anglais et leur flegme). Ce système, pourtant, perdait rapidement de sa millénaire légitimité sous les coups de la rationalité des nouveaux savoirs scientifiques et ses présupposés holistes ne pouvaient persister que sous la forme de représentations locales et populaires.

On peut pourtant se plaire à chercher encore quelque chose de ces anciennes formules dans la nouvelle sensibilité dont s'entoure le rapport entre «peuple » et «territoire » (Corbin, 1988 et Thomas, 1988). Les eaux et les plantes, les montagnes et les plages, les déserts et les rochers, les steppes et les brumes, les oiseaux et les cailloux, le soleil brûlant et les nuits boréales - tout vient concourir à la construction d'un blason d'images nationales, instruit par les classifications et les mensurations savantes des naturalistes. Un nouveau totémisme se met en place, par la sélection d'éléments des séries naturelles pour définir le profil comparé des identités concurrentes dans une échelle planétaire. Pourtant, de très anciennes images glissent en-dessous de ces choix réfléchis, traduites par des rationalisations qui soumettent la nature à la tradition historique et culturelle. Le coq de la représentation nationale française ne s'est établi pour les mêmes raisons classificatoires

12. Au Brésil, on dispose de l'exemple précoce du gouvernement hollandais au Nordeste brésilien, où fleurit - sous le Prince Jean-Maurice de Nassau-Siegen (1604-1671) - une administration aussi soucieuse de l'efficacité économique et politique que de la dimension scientifique et artistique de la domination. Que l'on songe aux oeuvres de Franz Post et Albert Eckhout, toujours évoquées comme des témoignages de la bonne alliance entre le raffinement de l'art européen et la fascination de la nature brésilienne. 
que celles du ip $\hat{e}^{13}$ jaune pour la nation brésilienne. Ni la présence de l'arbousier dans le drapeau de la ville de Madrid s'est imposée par les mêmes voies symboliques que le cèdre pour la représentation nationale libanaise. Dans tous les cas, pourtant, il s'agît de signes locaux d'une même activité complexe et systématique d'investissement sur le potentiel de symbolisation des signes naturels - et elle se nourrit de plus en plus des séries organisées par les nouvelles procédures scientifiques.

Un beau monument de la transition entre l'ancienne imagerie physico-morale et les nouvelles corrélations entre nation et nature est le livre sur le rapport entre la Suisse et les Alpes, écrit en 1732 par l'éminent physiologiste Albrecht Von Haller. On y trouve, en même temps, l'arrière-fonds traditionnel des correspondances humorales entre la terre et ses gens et l'éloge spécifique du paysage (les hautes montagnes : leur isolement, leur grandeur, leur défi) et de son « influence » sur la constitution morale des citoyens de la petite république (Schama, 1995). Il faut garder à l'esprit combien l'imaginaire des Alpes, des forêts tempérées et des tempêtes de neige, est resté au centre des fantaisies les plus sentimentales du romantisme germanique. Le nazisme a su reprendre ce fonds imaginaire avec une rare habileté, ce dont témoigne la mémoire du «Nid des Aigles » de Hitler à Garmisch-Partenkirchen.

C'est toujours Simon Schama qui évoque la série de nouvelles institutions auxquelles incombe au $19^{\mathrm{e}}$ siècle représenter cette association entre nature et nation. La ville de Londres est le premier laboratoire : on y fait inaugurer en 1820 un jardin zoologique; en 1846, la grande serre publique de Regent's Park, et - finalement - en 1851, le Crystal Palace abritent celle qui est considérée comme la première Exposition Internationale (Stocking, 1987). Sous son étonnante coupole en fer, les dernières nouveautés de l'industrie triomphante côtoient les merveilles de l'agriculture à grande échelle et les trésors d'une nature finalement domptée. Les Etats-Unis suivent rapidement l'exemple : le Central Park de New York est inauguré en 1857, comme une grandiose synthèse entre la nature et l'artifice urbain. Le Yosemite Valley est classé comme un «monument national » en 1864, comme le premier exemple de la formalisation du concept de préservation de la nature en unités géographiques discrètes censées représenter la richesse morale de la nature - sous l'égide du paysage (Schama, 1995 : 17).

Cette réorganisation des valeurs attribuées à la nature en échelle planétaire se fait accompagner des nouveaux développements de la médecine, dès le début du $19^{\mathrm{e}}$ siècle surtout de la médecine préventive et sanitaire, bientôt succédée par le mouvement hygiéniste. Une vaste entreprise de formalisation d'un « panorama » de la Terre se met en route, par la voie de la géographie et de la climatologie émergentes, liées aux investigations sur les fièvres et les « maladies tropicales », ainsi que sur la spécificité de la reproduction humaine dans les différentes « régions » de la planète. Ces investissements suivent de près, évidemment, le rythme accéléré du colonialisme, aussi intéressé à l'exploitation des ressources natives qu'à la survie des populations consommatrices et des individus représentant des intérêts du commerce et de l'industrie.

Ce n'est pas par hasard que ce soit la botanique la science naturelle qui se développe le plus intensément au $19^{\mathrm{e}}$ siècle. On en attendait aussi bien de nouvelles ressources pour les

13. Tabebuia chryssotricha, arbre de la famille des bignoniacées, fréquent sur les forêts de la côte atlantique, réputé pour ses fleurs d'un jaune intense et pur. 
réseaux marchands ${ }^{14}$, que des moyens pratiques de contrôle des maladies et des épidémies. Von Martius nous a légué, dans ce sens, un Systema Materiae Medicae Brasiliensis, à côté de sa grande description taxonomique et phytogéographique ${ }^{15}$. On ne peut pas oublier, d'autre part, l'importance de la quête de nouvelles sources de satisfaction sensorielle, déclenchée dès la Renaissance dans le monde occidental, comme le démontre la mode de certaines fleurs et son immense intérêt économique (l'importance du marché des tulipes au $16^{\mathrm{e}}$ siècle n'en est qu'un exemple parmi d'autres) (Elliot, 2003).

L'immense valeur imaginaire des «tropiques » se condense pendant cette période, à cheval sur les fantaisies de l'évolutionnisme social naissant, de la médecine préventive, des sciences naturelles descriptives et du capitalisme colonial. L'image commune à la Renaissance des continents représentés par quatre belles femmes parées de leurs symboles spécifiques et croisés de nature et de civilisation disparaît progressivement au profit d'un régime d'oppositions binaires entre civilisation et barbarie, qui se redouble dans l'opposition entre une nature tempérée - souple et accueillante - et une nature tropicale - hostile et incontrôlable ${ }^{16}$.

Ce nouveau cadre imposait à chaque nation un travail imaginaire propre, à la mesure des références empiriques et des moyens culturels disponibles en chaque cas. Ce qui s'imposait inévitablement était l'élaboration d'un récit symbolique plausible et susceptible d'être offert sur le nouveau marché des images internationales qui s'amorçait.

La création, à la fin du $18^{\mathrm{e}}$ siècle, d'un service de porcelaine où chaque pièce présente le dessin d'un exemplaire de la végétation d'un petit pays du Nord de l'Europe est encore aujourd'hui un témoignage précieux du début de ce processus de "nationalisation » de la nature. Le service de la Flora Danica est toujours exposé à Copenhague comme un des symboles de l'enracinement profond de la nation danoise à son territoire, où la continuité naturelle vient souligner et soutenir son long parcours historique. Cette fonction exemplaire exigera bientôt davantage que des pièces d'apparat pour les cours royales. Il faudra permettre que ces images puissent se répandre et se laisser consommer par l'ensemble de la population, en même temps que d'autres ressources symboliques d'un patriotisme renouvelé. Les musées d'histoire naturelle auront un rôle primordial dans ce processus.

14. La circulation des espèces botaniques sur la planète s'était intensifiée énormément dès le $16^{\mathrm{e}}$ siècle. L'histoire économique moderne abonde en exemples des effets extraordinaires de cette circulation. Le cas du Brésil est particulièrement intéressant. La canne à sucre, d'abord acclimatée dans ses îles atlantiques par les Portugais, devint la première cause de l'essor économique de la colonie. Le café, dont les premières plantes ont été passées en contrebande des colonies françaises au début du $19^{\mathrm{e}}$ siècle, devint le moteur de l'articulation de l'économie nationale aux marchés internationaux après l'indépendance. L'économie de l'Amazonie, florissante au début du $20^{\text {e }}$ siècle grâce à l'exploitation du caoutchouc natif, plongea dans une rapide décadence après la transposition de cette plante dans les domaines anglais de l'Orient. La circulation internationale des espèces botaniques a suscité des analyses qui soulignent son caractère essentiel pour la compréhension de la culture occidentale moderne (surtout, Sahlins, 1996).

15. L'intérêt pour les espèces tropicales a suscité très tôt l'organisation de jardins d'acclimatation, qui se sont multipliés un peu partout au long des $18^{\mathrm{e}}$ et $19^{\mathrm{e}}$ siècles. Le jardin botanique de Rio de Janeiro, crée en 1808 , peut être considéré comme la plus ancienne institution scientifique du pays.

16. Une riche analyse de la dimension parallèle des représentations européennes sur l'âme des indigènes des colonies américaines aux premiers siècles de la Conquête peut être trouvée dans Viveiros de Castro, 1993. 
On pourrait opposer à mon argument le fait que les grands musées métropolitains d'histoire naturelle ne se soient jamais restreints à la présentation de leurs respectives « natures nationales ». On n'aurait pas tort; puisque le prestige des nations métropolitaines ne s'est pas bâti sur leurs seules richesses et merveilles propres, mais sur leur capacité de représenter l'universalité du monde atteint par leur pouvoir. Cette disposition universaliste a été renforcée sans doute par la condition de puissance coloniale; mais elle n'en dépendait pas. Il s'agissait surtout d'être capable d'exprimer les valeurs de l'universalité scientifique en même temps que les valeurs de la particularité nationale. Cette procédure permettait une sorte de légitimation universaliste du particularisme. Vers la fin du $20^{\mathrm{e}}$ siècle, on pouvait encore admirer de très beaux et très anciens dioramas sur les écosystèmes de la Nouvelle Angleterre au milieu de plusieurs nouvelles expositions sur des sujets de science universelle.

Un exemple très caractéristique est celui du météorite qu'on appelle Bendegó et qui est toujours dans une place d'honneur dans l'exposition du Musée National de Rio de Janeiro. Il s'agit évidemment d'une pièce « naturelle », extra-planétaire d'ailleurs, exemplaire de plusieurs processus et réseaux d'informations scientifiques d'une valeur universelle. Sa présentation (et représentation) publique relève pourtant d'un régime de valeurs qui renvoie plutôt à son poids exceptionnel, à sa monumentalité et à sa "nationalité » exemplaire. L'histoire édifiante de son transport de l'intérieur de la province de Bahia jusqu'à la capitale de l'Empire vers la fin du $19^{\mathrm{e}}$ siècle (1888), la permanence de l'épithète de la localité où on l'a trouvé, sa présentation traditionnelle dans le hall d'entrée du Musée sur trois socles sculptés en marbre dans le style néo-classique et chargés d'inscriptions commémoratives, tout permet de comprendre l'intense investissement symbolique dont de tels objets ont été partout entourés - et combien ils ont pu servir aux fins de la morale publique nationale en formation.

6. S'il est vrai qu'on peut parler de la production d'une « nature nationale », il est

6. aussi bien possible de le faire à propos d'une «culture naturelle », qui vient compléter le réseau d'images nécessaires au défi des identités nationales à produire.

Une des dimensions fondamentales de la justification romantique des nations modernes est celle du rapport instaurateur, originaire, entre territoire et population (y inclus sa culture). Les attentes d'une solidarité holiste exprimées par cet idéal se fondaient sur la longue convivialité censée avoir soudé ces deux éléments, dans le sens de ce qu'on est venu à appeler une « tradition nationale». La plus grande partie de ce qu'on appelle aujourd'hui les « sciences humaines »-d'abord des « sciences morales » ou « de l'esprit » (Geisteswissenschaften) - a vu le jour ou s'est progressivement distinguée de cette grande nébuleuse : archéologie, histoire, géographie humaine, philologie/linguistique, anthropologie, psychologie sociale, théorie littéraire etc.

Pour ce qui concerne notre thème principal, il faut souligner que les musées d'histoire naturelle ont dû ainsi couvrir non seulement les séries informatives de la nature au sens strict, mais aussi les séries «culturelles » qui s’y sont associées au sens large - dans un jeu de continuités essentiel à la naturalisation des nations.

Ce processus a été particulièrement critique en ce qui concerne les informations relatives aux conditions de l'expérience humaine considérées comme « traditionnelles », « autochtones », « natives » ou «primitives ». A la lumière de la formule de Taine $\mathrm{du}$ rapport essentiel entre l'homme et son «milieu », se mettait en jeu une complexe négociation entre la légitimité de ces conditions en tant que témoignage d'un temps 
«primordial», originaire, et leur illégitimité en tant que témoignage d'un temps " primitif », arriéré17. La grille évolutionniste, première grande expérience d'imposition d'une rationalité universelle aux données spécifiques de l'expérience humaine, venait ainsi s'opposer à la valeur particulariste de l'enracinement des peuples ${ }^{18}$.

Certains domaines de savoir sont devenus particulièrement exemplaires de ce développement, ce qui leur a valu une place prééminente dans les musées d'histoire naturelle : l'archéologie, l'ethnologie et l'anthropologie physique étaient très bien douées pour représenter les rapports originaux de l'humain à la nature, d'autant plus qu'elles pouvaient offrir aux démonstrations muséographiques une pléthore d'objets de haute expressivité.

La France au tournant des $18^{\mathrm{e}}$ et le $19^{\mathrm{e}}$ siècles concentre de beaux témoignages de la complexité de ce processus historique. Le projet des Idéologues de production d'une connaissance universelle sur l'humain qui s'est concentré dans la Société des Observateurs de l'Homme (qui a pris, à son tour, l'initiative d'organiser l'Expédition Péron, partie vers les mers du Sud avec des instructions de recherche anthropologique formulées par Buffon et par le Baron De Gérando - (Jamin, 1979 et Gusdorf, 1974)) est la contrepartie civile de la manière par laquelle Napoléon Bonaparte avait incorporé à ses grands mouvements militaires des équipes de recherche scientifique. Les résultats de l'invasion de l'Egypte comptent encore de nos jours plutôt par leurs résonances scientifiques que par leur dimension géopolitique $^{19}$. Au long du $19^{\text {e }}$ siècle la plupart des expéditions scientifiques couvrait - parfois sous la responsabilité du même chercheur - aussi bien les séries d'information strictement naturelle que les séries d'information culturelle. Dans le cas du Brésil, c'est suffisant d'évoquer l'importance des informations et hypothèses anthropologiques dans le travail de Von Martius ou de Charles Frederic Hartt pour témoigner de cet englobement de la « culture naturelle» par la « nature nationale ».

7 La grande institution « primordiale » chargée du culte de la « nature nationale » 7. au Brésil a été le Museu Nacional. D'abord solitaire et à vocation universelle, à partir de sa fondation sous le nom de Musée Royal en 1818, il s'est vu partager les fonctions de représentation de la nation successivement avec l'Institut Historique et

17. Dans la langue allemande, on a pu jouer avec l'usage des deux préfixes $U r$ - e Natur- (respectivement « originaire » et « naturel ») pour exprimer toute la gamme des valeurs positives et négatives attribuées aux différentes conditions et dimensions de l'histoire de l'humanité (peuple, religion, langue, coutumes etc.).

18. A côté de cette transformation en « histoire naturelle » de toutes les dimensions des phénomènes culturels qui se distinguaient de la « civilisation » occidentale, on peut voir en cours - au long du $19^{\mathrm{e}}$ siècle - un autre type de réduction, plus strictement universaliste, qui considérait comme strictement «physiques » tous les phénomènes de la « nature humaine ». Considérée comme un simple épiphénomène du substrat physique, corporel, l'expérience humaine devrait être progressivement éclairée par le progrès de la science. Cette conception - dont on voit un renouveau certain à la fin $\mathrm{du} 20^{\mathrm{e}}$ siècle - a eu les plus graves influences sur le développement de la culture occidentale. On se bornera ici à souligner certains aspects de sa présence dans l'oeuvre de représentation nationale en cours dans les musées d'histoire naturelle.

19. Un des grands monuments de l'histoire des sciences modernes - et, en plus, de l'histoire des arts - est la Description de l'Egypte (1830), avec ses magnifiques planches naturalistes, artistiques et ethnographiques. « Il arrivera que l'ouvrage de la Commission des arts excusera aux yeux de la postérité la légèreté avec laquelle notre nation s'est, pour ainsi dire, précipitée en Orient.» (Lettre de Geoffroy Saint-Hilaire à Cuvier, du 27 novembre 1799). 
Géographique Brésilien, les premières Facultés de Droit et de Médecine ${ }^{20}$, l'Académie de Médecine, l'Académie Impériale des Beaux-Arts et une série d'autres institutions postérieures. Même la fonction de «musée d'histoire naturelle », qui s'était précisée lentement, a du être partagée avec le Museu Paulista (créé dans les années 1890) et avec le Museu Paraense (créé dans les années 1870 et restructuré par Emilio Goeldi dans les années 1890), au début de la période républicaine. L'avènement de toutes ces autres institutions est à la fois un signe de la lente structuration d'une administration nationale complexe et moderne au Brésil et des difficultés et vicissitudes du projet même d'un image nationale disponible à l'intérieur et à l'extérieur du pays (Lopes, 1997 et Santos, 2000).

Les premières expositions publiques ont été ouvertes encore au début des années 1820, présentant des séries « naturelles » et de « civilisation » (objets d'art, tableaux, machines etc. ${ }^{21}$. Les collections se sont enrichies à un rythme irrégulier avec les dépôts d'objets ex officio et d'une partie des résultats des expéditions de voyageurs étrangers, qui se sont multipliées après l'ouverture des ports en 1808. Les fonds de cette période héroïque présentaient une extrême variété. Une importante collection exemplaire de minéralogie (Collection Werner), acquise par la Couronne portugaise en Allemagne représentait le coté plus systématique et scientifique de cet éventail d'objets. C'est possible qu'une petite partie des animaux empaillés qui avaient été présentés au public de Rio de Janeiro dans le petit musée colonial qu'on appelait la Casa dos Pássaros fut encore disponible pour ces nouvelles expositions, après un long dépôt dans l'Académie Militaire Royale. Une partie des collections royales venues de Lisbonne, des tableaux et des objets de prestige surtout, avait été prêtée au Musée. On fait finalement allusion aussi à des machines, présentées au public dans ce mélange d'intérêt scientifique, de disposition technologique et de respect pour les « forces de la nature » qui caractérisaient la culture occidentale moderne dès le début du $18^{\mathrm{e}}$ siècle. Tout autour de ces tableaux, machines et collections systématiques, s'étalaient les spécimens de la flore et de la faune locale, signe sensible de la « richesse » nationale, à cheval entre la réalité immédiate de la profusion tropicale et les souhaits d'un progrès économique soutenu ${ }^{22}$.

Une tension caractéristique de cette première période de la vie du Musée est celle de l'unité de représentation politique : l'Empire portugais, vaste et hétéroclite, ou le Brésil, cette colonie très spéciale devenue pour un temps la métropole même ? Les données sur ces premières expositions et les collections qui les soutenaient sont trop minces pour permettre d'approfondir cet aspect de la question. On peut la deviner entre les textes

20. Les premières facultés brésiliennes de Médecine ont été fondées à Bahia et Rio de Janeiro, en 1830 (après les chaires d'anatomie et chirurgie créés en 1808) et les premières facultés de Droit à Recife (Olinda, d'abord) et à São Paulo, en 1827, Cunha, 1980.

21. La capitale du Brésil passait à cette époque par l'influence modernisante des membres de la Mission Artistique Française appelée au Brésil en 1816. L'imagerie de l'Empire naissant a été fortement influencée par le travail de Jean-Baptiste Debret (1768-1848), ancien disciple de Jacques-Louis David et professeur à l'Académie Impériale des Beaux-Arts, créée en 1826. On lui doit, par exemple, le dessin du drapeau impérial brésilien (vert, comme ses immenses forêts; jaune, comme l'or de ses mines - dit-on encore) (Schwarcz, 1998).

22. «'Nature', then, was to be shown as a repository of species and primary sources, a catalogue of objects awaiting commodification, but also as a primordial and irreducible layer of Brazilianness, as the very 'soil' of nationality » (Andermann, s/d). 
juridiques programmatiques et les descriptions de quelques visiteurs. D'une certaine façon, cette tension a dû recouvrir au début l'opposition plus structurelle entre une vision plus universelle et scientifique et une autre plus particulariste et affective ${ }^{23}$. On enregistre les fréquentes critiques des visiteurs étrangers sur la prétention plus générale du Musée et la faiblesse de la représentation de la nature locale (Andermann, s/d).

Une autre dimension générale qu'on ne peut pas oublier de la période initiale du Musée est le fait qu'un musée à prétention universelle à cette époque hérite encore d'une manière très intense de la représentation du « cabinet de curiosités » et - dans le cas d'un musée royal - de l'image d'un « trésor national ». Le 1 ${ }^{\text {er }}$ Empire au Brésil est d'autant plus proche de cette condition, que l'Empereur Pedro I incarne encore très nettement le modèle dynastique traditionnel de l'Occident, avec sa dimension hiérarchique et personnaliste générale soutenue dans son cas par une personnalité fougueuse et aventurière ${ }^{24}$.

Certains aspects des collections du Musée relèvent de ce rapport original avec la dynastie et ses propriétés symboliques de Maison Royale. Le premier cas est celui de la collection de l'Ancienne Egypte, dont le noyau consiste dans une considérable série de pièces présentée, comme des curiosités précieuses, au port de Rio de Janeiro par un marchand italien. L'Empereur prît la décision personnelle d'acquérir cette collection et de la léguer au Musée. La collection fût enrichie plus tard par le legs au Musée d'autres pièces que son fils, Pedro II, avait reçu en cadeau du khédive d'Egypte lors de sa longue visite aux centres archéologiques du Proche-Orient. Un autre exemple frappant est celui de la collection Gréco-Romaine, qui consiste en une importante série d'objets emportés au Brésil, comme partie formelle de sa dot, par la fille du Roi de Naples, Thérèse-Christine de Bourbon-Siciles, devenue impératrice par son mariage à Pedro II. La princesse ellemême avait subvenu à des fouilles importantes dans une de ses propriétés au Sud de l'Italie, ce qui rendait sa collection - devenue brésilienne - en même temps un exemple de l'identité dynastique (avec des trésors symboliques liés à la tradition historique et à certains territoires), de l'identité nationale (alliance par mariage entre les chefs d'Etat de deux nations) et de l'identité scientifique (témoignage d'un intérêt savant, méthodique, pour l'archéologie et l'histoire).

Ce curieux mélange entre intérêt culturel, savant, et intérêt politique national a été constamment et directement en jeu dans l'histoire initiale de l'institution. Un autre exemple plein d'intérêt, est celui du manteau royal des îles Sandwich, dont un prince local en voyage avait fait cadeau à l'Empereur. Il est bien probable que plusieurs pièces des collections du Musée aient été utilisées pour des échanges de ce genre. Un cas toujours très cité est celui de l'utilisation des exemplaires empaillés de toucans de la collection ornithologique pour composer avec les plumes jaunes de leur poitrine la pèlerine du manteau officiel du sacre de l'Empereur Pedro II - au lieu de l'hermine de la tradition royale (et naturelle) européenne.

23. Le rapport entre une vision universaliste et une vision « impériale » n'est évidemment pas linéaire; mais la discussion sur l'imagerie de l' « empire » en Occident et le processus de rationalisation du monde serait ici tout à fait pertinente, d'autant plus qu'il concerne la conception même de l'Eglise catholique aussi.

24. Le prince Pedro, promoteur de l'indépendance et premier empereur du Brésil, est plus tard devenu le champion des réformes libérales au Portugal et couronné roi (Pedro IV). Son nom a fait partie de la liste des possibles occupants du trône grec, après 1827 , lorsque le processus d'indépendance de la Grèce fut reconnu par les grandes puissances. 
La professionnalisation des fonctions du Musée, selon le modèle rationnel qui s'organisait dans les centres métropolitains ne s'est produite que très lentement, au gré des rapports entre le personnel scientifique local et l'évolution scientifique internationale, de la force relative des tendances modernisatrices dans l'Etat et des crises financières successives de l'Etat. Une première grande réforme administrative a eu lieu dans les années 1830, sous l'administration de Frère Custódio Serrão ${ }^{25}$. Une autre grande vague de modernisation a eu lieu dans les années 1870, sous l'influx de l'échange régulier avec l'étranger et en réponse aux nouvelles demandes de rationalité de l'administration publique et de promotion de l'éducation et de la vie intellectuelle qui se sont imposées dans le mince monde urbain brésilien vers la fin de la Guerre du Paraguay ${ }^{26}$.

Le Musée est le siège d'une série d'initiatives importantes liées au développement institutionnel du pays au long du $19^{\mathrm{e}}$ et du $20^{\mathrm{e}}$ siècles. En dépit de sa courte vie, on ne peut manquer de citer la création, au sein du Musée, de la Société d'Aide à l'Industrie Nationale (Sociedade Auxiliadora da Indústria Nacional, 1825), qui annonce, très tôt, cette vocation de carrefour entre les valeurs de la nation, de la nature et du progrès.

Une des dimensions de ce rôle stratégique est la participation de l'institution dans ces efforts épiques de connaissance scientifique et contrôle politico-administratif que constituent les expéditions d'Etat, dans un pays avec un territoire si vaste et si mal connu et intégré. Le propre musée a commencé à entreprendre des expéditions scientifiques à partir du milieu du siècle, quand Francisco Raimundo de Faria a été envoyé chercher pour l'institution des « produits naturels et ethnographiques de la vallée de l'Amazone » (Castro-Faria, 1998 : 62). Le premier grand exemple d'une expédition officielle du nouvel Etat brésilien est, pourtant, celui de la Commission Scientifique d'Exploration, connue comme Comissão do Ceará, ou comme Comissão das Borboletas, dont les matériaux seront mis en dépôt surtout au Musée (spécimens, rapports, bibliothèque, gravures etc.). Les travaux de l'équipe se sont déployés au Nordeste du Brésil, autour de la Province du Ceará, entre 1859 et 1861 . L'important poète romantique Gonçalves Dias en faisait partie $^{27}$ et l'échantillonnage de la nature des provinces du Nord a été accompagné d'une vaste description iconographique d'objets des cultures indiennes de la région.

Le Musée a participé, de manière directe ou indirecte, de toutes les autres grandes expéditions nationales. On ne peut que faire une brève mention aux travaux de Charles Frederic Hartt, géologue du Musée, en tête de la Commission Géologique de l'Empire (1875-1877) (Freitas, 2001); à ceux de Cândido Mariano Rondon, premier directeur du

25. «Under the direction of Frei Custódio Alves Serrão, a former professor of chemistry at the Escola Militar, the institution was restructured in 1842, following the example of the British Museum (rather than the Muséum de Paris, which had until then provided the model): the collections were divided into four sections headed by subdirectors - comparative anatomy and zoology; botany, agriculture and mechanical arts; mineralogy and geology; numismatics, arts and customs -, and travelling naturalists - mostly of French or German origin - were contracted to supply the institution with species from the interior ». (Andermann, s/ d).

26. Les réformes de la fin du $19^{\mathrm{e}}$ siècle se font sous l'inspiration et le sévère contrôle du directeur Ladislau Netto (1870-1893), un typique représentant d'une illustre génération d'intellectuels avec une formation professionnelle précise engagé dans le processus de modernisation nationale.

27. On ne pourra pas développer ici les rapports de l'image de la nation et de « sa » nature dans la production littéraire brésilienne. Le thème est pourtant indissociable de notre objet, surtout à propos des auteurs romantiques du $19^{\mathrm{e}}$ siècle, où apparaît aussi clairement le passage par la « culture naturelle » des indiens et des types régionaux (voir, sur le « nativisme » romantique au Brésil, Schwarcz, 1998: 128). Tout aussi utile et stimulant aurait été un investissement homologue sur la peinture ou la photographie. 
Service national de Protection aux Indiens, en tête de la Commission Rondon (ancienne Commission des Lignes Télégraphiques du Mato Grosso et Amazonas, créée en 1890), qui s'est occupée, entre 1907 et 1915, de l'intégration pacifique des indiens du Brésil Central à la nation - et dont les matériaux seront incorporés surtout au Musée; ou aux successives expéditions d'un intérêt médical et sanitaire organisées par l'Institut Sorothérapique Fédéral (plus tard devenu l'Institut Oswaldo Cruz) à partir de 1900, comme celle de Belisário Penna vers les Etats du Nordeste vers 1916, avec la participation de Arthur Neiva (Directeur du Musée entre 1923 et 1926).

Une autre dimension cruciale de la fonction de représentation nationale est le rôle du Musée dans l'organisation de grandes expositions temporaires, soit à l'étranger, soit dans son propre espace institutionnel. Le Musée a été d'abord le responsable de la première exposition industrielle de Rio de Janeiro, en 1861, préparatoire de la première exposition nationale qui eût lieu la même année. Il s'est vu depuis successivement en charge des expositions de 1866, 1873 et 1875, préparatoires de la participation du pays dans les Expositions Mondiales (Castro-Faria, 1993 : 56-61; Schwarcz, 1998 : 385; Andermann, s/ d). En tous cas, il s'agissait de présenter les témoignages de la richesse naturelle nationale à côté des succès de l'agriculture et de l'industrie naissante. La participation du Musée dans la contribution brésilienne aux Expositions Internationales, dont la longue série avait été inaugurée par celle de Londres en 1851, lui a valu certainement une bonne partie de son prestige auprès de l'administration impériale. L'importance du rapport de la nation à ces expositions peut être jugée par la présence personnelle de l'Empereur à celle de Philadelphie, en 1876 (Schwarcz, 1998). C'est à partir de la $3^{\text {e }}$ Exposition, tenue à Londres, en 1862, que le Musée s'incombe systématiquement de l'organisation du pavillon brésilien, cette manifestation stratégique de l'image nationale. Une participation officielle éminente est enregistrée au moins jusqu'à l'Exposition Universelle de Chicago de 1893.

Un moment remarquable de ces fonctions de représentation nationale est celui de l'organisation de la première Exposition Anthropologique Brésilienne, en 1882 $\left(\right.$ Nascimento, 1991) ${ }^{28}$. Elle s'ensuivit à la première Exposition d'Histoire du Brésil, qui avait eu lieu à la Bibliothèque Nationale en 1881, et à la première Exposition de l'Industrie Nationale (1882), préparatoire de la participation brésilienne à l'Exposition Continentale Sud-Américaine de Buenos Aires, la même année. Le Directeur Ladislau Netto s'est occupé personnellement de l'organisation de cette grande exposition, dédiée à la connaissance des sociétés indiennes du territoire national. Un plan systématique d'obtention de collections représentatives des différentes traditions culturelles tribales a été mis en place plusieurs années en avance, avec la subvention de quelques expéditions spécifiques. On fit peindre des portraits et sculpter des images d'indiens par des artistes prestigieux et on fit venir même des représentants vivants de certaines tribus, qui s'installèrent dans le propre Musée ${ }^{29}$. L'Exposition suscita un vif intérêt public et fut visitée plusieurs fois par l'Empereur. Une bonne bibliographie s'est déjà occupée

28. Les musées (ou les expositions) d'ethnographie ne trouvent une place autonome que très lentement, par rapport aux musées d'histoire naturelle, dont ils sont généralement issus. La France ne met en place un musée de ce genre qu'en 1877 : le Musée du Trocadéro (Castro Faria, 1998).

29. Ce fonds constitue toujours une partie prestigieuse des collections et des expositions permanentes contemporaines d'ethnologie du Museu Nacional. 
des implications proprement savantes de l'Exposition, à propos aussi bien du statut des populations indiennes du Brésil (et de celui de leur étude systématique) que de leur place dans la structure de la nation (Castro-Faria, 1998; Nascimento, 1991; Schwarcz, 1998; Andermann, s/d).

L'avènement de la République, en soi, n'a pas beaucoup changé à ce rôle institutionnel du Musée. Un épisode important de l'histoire de cette époque doit être évoqué. Le Palais de Saint-Christophe, qui avait servi de résidence officielle de la Famille Royale et Impériale, dès l'arrivée de la Cour en 1808, a été offert par le Gouvernement Provisoire pour servir comme nouveau siège du Musée. Le transfert du Musée ne s'est achevé qu'en 1892, étant donné que la première Assemblée Constituante républicaine a dû y travailler jusqu'en 1891. On ne peut pas douter de la précision de la décision gouvernementale. On soulignait, d'abord, le prestige dont l'éducation et la science devraient jouir dans un régime fortement inspiré par le positivisme comtéen, en lui vouant un des immeubles les plus prestigieux de la nation. On se débarrassait en même temps de la mémoire d'un palais royal, dont la résistance idéologique monarchique aurait pu avoir fait un centre de révérence nostalgique. On rendait aussi indirectement hommage à l'Empereur déchu, assez populaire, qui s'était toujours distingué par son appui politique et intérêt personnel à la science et à l'érudition ${ }^{30}$.

L'importance de l'Exposition Anthropologique de 1882 et de la réflexion qu'elle suscita sur l'héritage indigène témoigne d'un tournant important des rapports entre nation et nature entre la fin du $19^{\mathrm{e}}$ siècle et la $2^{\mathrm{e}}$ Guerre Mondiale. Il s'agit de la prééminence des rapports entre civilisation et race ${ }^{31}$. Le Musée a encore une fois joué un rôle essentiel dans ce champ de débats politiques et scientifiques. J'ai déjà eu l'occasion d'exposer ailleurs la complexité de cette configuration idéologique (Duarte, 2000) et de ses développements dans l'histoire brésilienne. Ici, je ne peux qu'évoquer le rôle de João Baptista de Lacerda (directeur du Musée entre 1895 et 1915) dans les débats sur le rapport entre la civilisation nationale (qu'on jugeait insuffisante et qu'on tâchait de promouvoir) et le fait du métissage de la population brésilienne. Contre les idéologues du racisme et du dégénérationnisme (telles celles du Comte de Gobineau, qui avait été ambassadeur au Brésil et ami personnel de l'Empereur), se sont opposées des formules autochtones qui prônaient la possibilité d'une récupération nationale par un mélange de métissage « blanchissant $»^{32}$ et d'investissement à l'éducation.

30. Le Musée a hérité de plusieurs collections, spécimens, instruments et livres des membres de la famille impériale. Le premier cabinet d'herborisation brésilien a été probablement celui que l'Impératrice Léopoldine avait maintenu au Palais de St.-Christophe au début du $19^{\mathrm{e}}$ siècle. L'Empereur, son fils, travaillait dans un véritable cabinet savant dans ce Palais et avait fait installer un petit observatoire astronomique dans la terrasse au-dessus des salles d'apparat de la façade.

31. On ne peut pas oublier que la catégorie de « race » renvoyait justement à la 'nature' une bonne partie de ce qu'on peut classifier autrement comme de la « culture ». Les graves implications de cette dimension de la 'nature nationale' sont évidentes dans les politiques nationales de restriction de la citoyenneté par la race qui ont eu lieu surtout (mais pas exclusivement) dans l'entre-deux-guerres.

32. J. B. de Lacerda, spécialiste en anthropologie physique, est considéré comme un des principaux responsables de la théorie du «blanchissement progressif» de la population brésilienne. Une partie de ses efforts idéologiques s'est concentrée dans l'éloge et la promotion de l'immigration européenne pour contrecarrer l'influence africaine (Seyferth, 1989). 
Pendant les années 1920 et 1930 le Musée s'est encore distingué par sa contribution à la question de l'éducation nationale, notamment à travers l'action multiforme de Edgar Roquette-Pinto (Directeur du Musée entre 1926 et 1935) (Castro-Faria, 1998). On lui doit, par exemple, la création, au Musée, de la Revue Nationale d'Education, dont il fut l'éditeur des 21 numéros publiés. C'est une période, pourtant, où la professionnalisation des activités scientifiques s'intensifie, au détriment - évidemment - d'une plus immédiate subordination aux injonctions de représentation institutionnelle de la nation. On pourrait dire que la représentation se spécialise elle-même: le rôle primordial du Musée se manifeste dans des moments rituels importants pour l'histoire de la science nationale, comme dans le cas des visites de scientifiques importants comme Albert Einstein ou Marie Curie, qui se font photographier à côté du personnel local.

Cette visite assez sommaire à l'histoire du Museu Nacional de Rio de Janeiro dans le long processus de construction de l'identité nationale brésilienne n'a de sens ici qu'en tant qu'exemple de la configuration idéologique plus générale de la « nature nationale » qu’on a voulu esquisser comme un des traits saillants de notre culture occidentale moderne. En réalité, l'histoire des expositions - permanentes et temporaires - du Museu Nacional est presque entièrement à faire. Un travail important sur les archives du Musée a été entrepris récemment dans le cadre des études pour un grand projet de renouvellement de l'exposition permanente (qui devrait s'installer après la fin des travaux en cours dans les bâtiments) (Duarte \& Aranha Filho, 2003), mais il est encore largement insuffisant, étant donné la grandeur et la complexité de la tâche.

D'ailleurs, le défi du processus de renouvellement des expositions de l'institution se heurte toujours - parmi d'autres enjeux - à la question du rapport entre l'universalisme de la science et la particularité de la nation (ou des intérêts locaux). Plusieurs solutions importantes du projet qui vient d'être dessiné à l'intérieur de l'institution répondent directement au souci de garder un certain équilibre entre ces deux pôles, tout en préservant la prééminence de la visée scientifique - essentielle pour l'identité contemporaine de l'institution (Duarte \& Aranha Filho, 2003). On pourrait parler donc d'une considérable permanence du problème de la «nature nationale», en dépit des déplacements institutionnels, stratégiques et scientifiques qui se sont imposés après la $2^{\text {e }}$ Guerre Mondiale.

Q Il y aurait beaucoup à dire sur les avatars plus récents de la notion de « nature 8. nationale ». On se borne ici à évoquer quelques directions possibles, facilement visibles en action dans la vie nationale contemporaine et même des institutions vouées à la connaissance de la « nature » (comme le sont toujours les musées d'histoire naturelle).

La première direction est celle du renforcement de la dimension marchande, économique, du nœud imaginaire de la nature par rapport à la nation. Ce processus s'impose d'abord par un déplacement de la nature visible, sensible, vers les dimensions plus abstraites de la génétique, de la pharmacologie ou de la biochimie. D'autre part, s'impose d'une manière plus évidente ou formelle le conflit entre l'intérêt des économies nationales et ceux du marché international (forces multinationales, par exemple). Ce conflit a toujours été présent dans l'histoire moderne ${ }^{33}$, mais le caractère plus abstrait ou

33. L'histoire du Brésil est particulièrement riche en exemples de l'importance économique et politique de la circulation des espèces naturelles, comme on a évoqué ici à propos de la canne à sucre, du café et du caoutchouc. 
subtil des matériaux en jeu impose de nouvelles formules à la guerre technologique. Au fond du conflit restent très visibles, pourtant, les enjeux de la nature nationale, comme en témoignent les développements juridiques très inquiets et très controversés qui entourent la protection nationale (et même, parfois, ethnique ou culturelle) des droits au patrimoine génétique ou à l'exploitation biomédicale de substances intrinsèques aux espèces naturelles. On se déplace davantage, ici, sans doute, de la dimension de l'image de la nature vers la dimension de sa « richesse ». L'image national reste pourtant toujours présent dans de représentations telles que la menace de la perte du droit d'usage économique de certaines substances naturelles considérées comme typiquement « nationales ».

La seconde direction découle de la récente métamorphose de la «nature» des naturalistes en «biodiversité » et en «écologie». On accentue davantage dans ce processus l'importance des aspects de la vie naturelle liés aux destins de la propre civilisation et de son constant progrès technologique et la conscience des risques de déstabilisation du rapport positif entre connaissance de la nature et satisfaction humaine qui a caractérisé notre tradition culturelle (Thomas, 1988). Ce déplacement intervient sur les représentations nationales à une échelle planétaire, étant donné la croissante nécessité d'établir des conventions internationales pour la préservation des ressources naturelles et des conditions positives du climat de la Terre. L'émergence de ces préoccupations n'est évidemment pas récente, mais plutôt sa prééminence sur les question de connaissance directe de la vie naturelle. La valeur stratégique de la connaissance classificatoire biologique classique (l'entreprise linnéenne qui caractérise toujours surtout la pratique de la biologie dans les musées d'histoire naturelle) se trouve ainsi déplacée, sous le nouveau signe de la « biodiversité », en réponse à des défis plus systémiques et hautement conscients de sa qualité politique (Castro, 2003) ${ }^{34}$.

Le déplacement de la notion scientifique de nature au long du $20^{\mathrm{e}}$ siècle est certainement à prendre en compte. Le développement des sciences humaines modernes, l'avènement d'une physique consciente des limites de la connaissance humaine, l'épaississement du monde biologique après la découverte de nouvelles couches et dimensions des processus vitaux, tout a contribué pour que le fétichisme de la nature se mue en fétichisme de la propre science - on pourrait dire. Même si la question s'était déjà posée au $19^{\mathrm{e}}$ siècle (surtout de la part des nations en régime de changement et croissance accélérée, tels les Etats-Unis, l'Allemagne ou le Japon), il faut reconnaître que la question d'une « science nationale » $\mathrm{s}$ 'impose fortement et tend à occuper le devant de la scène pendant la période fortement nationaliste de l'entre-deux-guerres. Une curieuse contradiction oppose une activité scientifique toujours plus abstraite et universaliste et une croissante soumission aux enjeux de la lutte internationale, dont l'essentielle dimension scientifique de la $2^{\mathrm{e}}$ Guerre Mondiale est un important cas de figure.

Le Brésil serait également sensible à ce déplacement - à un niveau plus discret. La fondation de l'Académie Brésilienne de Sciences, en 1916, est certainement un des signes d'un processus plus complexe, qui a dû faire attention aussi au niveau de la politique intérieure : celle de l'opposition des régions ou provinces à l'intérieur de la nation.

34. La question de la conservation de la nature est fortement liée à une autre dimension symbolique importante qu'on n'a pas pu développer ici : son caractère de source de satisfaction sensorielle. La création du tourisme moderne, avec sa panoplie d'images concernant les valeurs de la nature et de la civilisation, intervient d'une manière cruciale sur les stratégies identitaires des nations, d'autant plus que certains sports et presque toutes les activités de loisir dépendent du 'trésor' naturel national. 
La création du Museu Paulista au début de la période républicaine avait déjà annoncé très clairement cette dimension des rapports entre nation, science et nature. Son fondateur, Hermann Von Ihering, avait souligné très clairement son intention de créer une institution « purement » scientifique (par opposition à une institution où cette intention était souillée par la dimension de représentation nationale, comme le Museu Nacional) (Andermann, s/ d). En effet la prétention idéologique s'accordait heureusement au dynamisme économique très particulier de la province de São Paulo et aux aspirations de son élite de constituer un pôle culturel alternatif à celui de la capitale fédérale ${ }^{35}$.

On ne peut pas ignorer que la question de la "nature nationale ", elle-même, se déplace vers des dimensions toujours plus subtiles et sublimées - pour ainsi dire. On a fait mention à sa concentration sur le thème de la race, civilisation et éducation nationales entre les années 1920 et 1940, surtout au Brésil. En effet, tout le développement des sciences humaines dès cette époque témoigne de la même tension originelle entre l'ambition universaliste de la pensée et la condition locale, particularisée, des matériaux qui se présentent à l'analyse. L'injonction est évidemment plus forte dans le cas des faits de société et de culture, inséparables de la condition « nationale » de sa naissance. Le Museu Nacional a vu se développer dans son département d'anthropologie, à partir des années 1960, un programme d'études et recherches en "anthropologie sociale » qui s'est vite constitué comme un des centres les plus prestigieux de sa spécialité. On aurait certainement pu examiner la série des intérêts et des polémiques scientifiques qui s'y sont déployés pendant les dernières quarante années à la lumière du thème de la " nature nationale », soit dans l'intérêt comparatif des grands processus de changement dans la campagne et dans les villes brésiliennes, soit à propos des questions d'identité ethnique, régionale et nationale, soit à propos des différents styles d'analyse des cultures tribales (ou de son rapport à la nation), soit à propos des manières locales de produire une vie religieuse, politique ou intellectuelle ${ }^{36}$.

Les déplacements se sont produits aussi du côté des sciences naturelles. Une grande tension oppose les scientifiques dédiés aux activités taxonomiques et ceux qui se sont tournés directement vers les processus sous-jacents à la vie naturelle. L' hégémonie de cette dernière alternative dans le système scientifique national limite fortement le rôle et l'activité des musées d'histoire naturelle, contraints à s'occuper, à la fois, des dimensions plus mécaniques des collections naturalistes et des défis plus poussés de la science biologique contemporaine. Dans le cas du Museu Nacional, on peut réfléchir au fait que la seule salle nouvelle d'exposition permanente ouverte entre la fin des années 1960 et le début des années 1990 a été axée sur la « biodiversité »- un concept de transition fort stratégique pour les « naturalistes » biologistes.

35. Ce processus n'a jamais cessé de constituer une des caractéristiques dynamiques de la vie culturelle du Brésil. En ce qui concerne le champ scientifique, la puissance des universités et instituts scientifiques de l'Etat de São Paulo et de sa riche fondation locale d'appui à la science (la FAPESP a été créée en 1962) est un des éléments majeurs de la présente conjoncture nationale. Un exemple curieux des rapports de cette dimension régionale du problème de la «nature nationale » est le fait que l'Etat de São Paulo est le seul à avoir mis en place, à la fin du $20^{\mathrm{e}}$ siècle, un système universel de description de la flore et de la faune locales (Projet Biota - São Paulo, FAPESP), qui suscite l'envie de tous ses voisins et des autorités nationales.

36. L'œuvre de Mariza Peirano présente une réflexion stimulante sur la dimension « nationale » de la formulation des problèmes des sciences sociales au Brésil (Peirano, 1981, 1998). 
On pourrait finalement parler d'une dernière nouvelle direction, plus localisée. Il s'agit de la croissante importance imaginaire (ou popularité) des sciences historiques de la nature, notamment de la paléontologie. Une bonne partie du succès narcissique national des séries archéologiques et ethnographiques, qui avait atteint son sommet vers la fin du $19^{\mathrm{e}}$ siècle, s'est déplacée vers les séries des faits paléontologiques. Ce déplacement a, en réalité, concerné surtout la connaissance des paleo-vertébrés, sous la vogue des « dinosaures » qu'on a pu attester vers la fin du $20^{\mathrm{e}}$ siècle. On y voit en action toujours la dimension sensible, disponible au totémisme, de la série des espèces naturelles (en dépit des croissantes difficultés techniques de cette notion dans la biologie contemporaine) qu'on pourrait classifier comme de « grandeur nature ». Mais on la voit, significativement, déployée sur le temps, douée d'une épaisseur chronologique bien plus profonde que celle des faits de l'archéologie et de l'ethnologie - une sorte de mélange entre les propriétés symboliques de la biologie descriptive et de celles de la géologie. Pour ce qui nous concerne dans cet article, il faut souligner le fait que cette vogue vient aussi s'inscrire sur la dimension « nationale », avec la quête et l'éventuel éloge de l'antiquité ou richesse des gisements, des spécimens, des espèces ou des signes. On ne peut que souligner l'effet curieux que produit la juxtaposition des millions d'années caractéristiques des séries paléontologiques aux ambitions identitaires de nos nations modernes, âgées d'à peine quelques minces centenaires ${ }^{37}$.

Le thème de la «nature nationale », d'une si grande importance pour l'histoire moderne - politique et scientifique - est toujours vivant, présentant des cheminements et des défis qui concernent toujours et certainement les musées d'histoire naturelle et leur perplexité contemporaine - mais encore beaucoup davantage. Il est possible que le futur de ces institutions dépende de leur capacité à créer des nouvelles manières de contribuer aux demandes toujours présentes - mais bien plus subtiles et complexes - de bien servir aux ambitions de la raison et aux exigences du narcissisme collectif.

37. En l'an 2000, au cours des commémorations du $5^{\text {e }}$ centenaire de la 'Découverte du Brésil', un journal a présenté à ses lecteurs un tableau comparatif de l'âge des institutions culturelles brésiliennes, par le biais de l'âge des objets de leurs collections. Les séries géologiques et paléontologiques étaient évidemment imbattables, ce qui a valu au Museu Nacional encore une fois le palmarès de la représentation nationale. 


\section{Chronologie sommaire du Museu Nacional de Rio de Janeiro dans I'histoire du Brésil} (d'après la chronologie générale de l'institution établie par Jayme Aranha Filho)

\begin{tabular}{|c|c|}
\hline 1784 & $\begin{array}{l}\text { - Fondation, par le Viroi Luis de Vasconcellos e Sousa, à Rio de Janeiro, de la } \\
\text { Maison d'Histoire Naturelle (Casa dos Passaros) }\end{array}$ \\
\hline 1787 & $\begin{array}{l}\text { - Fondation, à Lisbonne, du Museu Real e Jardim Botânico do Sitio de Nossa } \\
\text { Senhora da Ajuda }\end{array}$ \\
\hline 1808 & $\begin{array}{l}\text { - Arrivée de la Cour Royale portugaise à Rio et ouverture des ports aux « nations } \\
\text { amies» } \\
\text { - Fondation du Jardin Botanique (d'abord Jardin Royal) de Rio de Janeiro }\end{array}$ \\
\hline 1817 & $\begin{array}{l}\text {-Arrivée à Rio de l'Archiduchesse Léopoldine, suivie d'une grande mission } \\
\text { scientifique officielle }\end{array}$ \\
\hline 1818 & •Fondation du Museu Nacional, à Rio, le 6 juin, par le roi Joao VI \\
\hline 1821 & $\begin{array}{l}\text { - Ouverture du Musée au public : six salles au total (quatre avec des spécimens } \\
\text { naturels au 2e étage; deux avec des machines au 1er étage) }\end{array}$ \\
\hline 1822 & - Indépendance du Brésil; début du 1er Empire (sous Pedro I) \\
\hline 1825 & $\begin{array}{l}\text { - Fondation, dans le cadre du Musée, de la Société d'Aide à l'Industrie Nationale } \\
\text { (Sociedade Auxiliadora da Industria Nacional) - qui va fonctionner jusqu'en } 1840\end{array}$ \\
\hline 1838 & $\begin{array}{l}\text { - Fondation de l'Institut Historique et Géographique Brésilien (Instituto Historico e } \\
\text { Geografico Brasileiro), dont les réunions se tiendront au siège du Musée }\end{array}$ \\
\hline 1842 & $\begin{array}{l}\text { - Première grande réforme administrative du Musée en vue d'une rationalisation } \\
\text { scientifique, sous le chimiste Frère Custodio Alves Ferrao }\end{array}$ \\
\hline 1844 & - Début du 2e Empire (couronnement de Pedro II) \\
\hline 1850 & $\begin{array}{l}\text { - Fondation, dans le cadre du Musée, de la société scientifique « Sociedade } \\
\text { Vellosiana de Ciências Naturais », qui publiera la revue Guanabara }\end{array}$ \\
\hline $\begin{array}{l}1859- \\
1861\end{array}$ & $\begin{array}{l}\text { - Expédition de la Commission Scientifique d’Exploration, connue comme } \\
\text { «Comissao do Ceara », ou comme «Comissao das Borboletas », dont les } \\
\text { matériaux seront déposés au Musée }\end{array}$ \\
\hline 1862 & - Exposition Internationale de Londres \\
\hline 1865 & - Visite de Louis Agassiz au Musée \\
\hline 1867 & - Exposition Internationale de Paris \\
\hline 1871 & $\begin{array}{l}\text { - Création d'un musée d'histoire naturelle à Belem do Para (qui deviendra plus tard } \\
\text { le Museu Paraense Emilio Goeldl) }\end{array}$ \\
\hline 1873 & - Exposition Universelle de Vienne \\
\hline
\end{tabular}




\begin{tabular}{|c|c|}
\hline $1875-1877$ & $\begin{array}{l}\text { - Travaux de la Commission Géologique de l'Empire, sous la direction de C.F. Hartt, } \\
\text { géologue du Musée }\end{array}$ \\
\hline 1876 & $\begin{array}{l}\text { - Deuxième grande réforme administrative en vue de la rationalisation scientifique, } \\
\text { sous le directeur Ladislau Netto (début de la publication des Archivos do Museu } \\
\text { Nacional, plus ancienne revue scientifique brésilienne encore active) } \\
\text { - Exposition Universelle de Philadelphie }\end{array}$ \\
\hline 1878 & - Exposition Universelle de Paris \\
\hline 1880 & $\begin{array}{l}\text { - Ouverture au Musée du Laboratoire de Physiologie Expérimentale, de J.B. de } \\
\text { Lacerda }\end{array}$ \\
\hline 1882 & $\begin{array}{l}\text { - Exposicao Antropologica Brasileira, sous le patronnage de l’Empereur } \\
\text { - Exposicion Continental Sud-Americana, Buenos Aires }\end{array}$ \\
\hline 1888 & - Arrivée au Musée du météorite de Bendegó \\
\hline 1889 & $\begin{array}{l}\text { - Exposition Universelle de Paris } \\
\text { - Proclamation de la République }\end{array}$ \\
\hline 1891 & $\begin{array}{l}\text { - Première Constitution républicaine, comme résultat d'une Assemblée } \\
\text { Constituante qui travailla au Palais de St-Christophe }\end{array}$ \\
\hline 1892 & - Transfert du Musée vers le Palais de St-Christophe \\
\hline 1893 & $\begin{array}{l}\text { - Fondation, à Sao Paulo, du Museu Paulista, sous la direction de Hermann Von } \\
\text { Ihering } \\
\text { - Exposition Universelle de Chicago }\end{array}$ \\
\hline 1894 & $\begin{array}{l}\text { - Emilio Goeldi devient directeur du Museu de Belem do Para et entreprend sa } \\
\text { modernisation }\end{array}$ \\
\hline 1900 & $\begin{array}{l}\text { - Réouverture de l'exposition permanente du Musée, dans son nouveau siège, avec } \\
\text { la présence du Président de la République }\end{array}$ \\
\hline $\begin{array}{l}1907- \\
1915\end{array}$ & $\begin{array}{l}\text { - Travaux de la Commission Rondon, sous la direction de l'officier de l'Armée } \\
\text { Candido Mariano Rondon (ancienne Commission des Lignes Télégraphiques } \\
\text { du Mato Grosso et Amazonas, créée en 1890), qui s'occupe de l'intégration des } \\
\text { Indiens à la nation - et dont les matériaux seront incorporés surtout au Musée }\end{array}$ \\
\hline 1920 & $\begin{array}{l}\text { - Ouverture au public de l'ancienne Salle du Trône du Palais de St-Christophe, } \\
\text { occupée par des matériaux historiques (numismatique) }\end{array}$ \\
\hline 1922 & $\begin{array}{l}\text { - Commémoration du premier centennaire de l'Indépendance; création du Musée } \\
\text { Historique National, surtout avec des fonds transférés du Musée }\end{array}$ \\
\hline 1924 & $\begin{array}{l}\text { - Fondation de l'Association Brésilienne de l'Education } \\
\text { - Visite d'Albert Einstein, qui plante un exemplaire de pau-brasil (bois-brésil) aux } \\
\text { jardins du Musée }\end{array}$ \\
\hline 1927 & - Visite de Marie Curie au Musée \\
\hline 1931 & $\begin{array}{l}\text { - Création du Service d'Assistance à l'Enseignement du Musée, dont le premier } \\
\text { responsable est Edgar Roquette-Pinto }\end{array}$ \\
\hline
\end{tabular}




\begin{tabular}{|l|l|}
\hline 1932 & $\begin{array}{l}\text { - Création, au Musée, de la RevistaNacional da Educaçao, ayant Roquette-Pinto } \\
\text { comme éditeur, dont } 21 \text { numéros ont été publiés }\end{array}$ \\
\hline 1938 & $\begin{array}{l}\text { - Le Palais de St-Christophe, siège du Musée, est classé comme monument } \\
\text { national par l'Institut du Patrimoine Historique et Artistique, qui vient d'être créé }\end{array}$ \\
\hline 1946 & $\begin{array}{l}\text { - Incorporation du Musée à l'Université du Brésil (aujourd'hui Université Fédérale de } \\
\text { Rio de Janeiro) }\end{array}$ \\
\hline 1947 & $\begin{array}{l}\text { - Réouverture au public, après une longue fermeture, des premières salles de la } \\
\text { nouvelle Exposition permanente, qui est toujours partiellement en place }\end{array}$ \\
\hline 1948 & $\begin{array}{l}\text { - Une des collections archéologiques « brésiliennes » du Musée (Collection Balbino } \\
\text { de Freitas) est classée comme monument national }\end{array}$ \\
\hline 1954 & $\begin{array}{l}\text { - Siège de la Première Réunion Brésilienne d'Anthropologie (création de } \\
\text { l'Association Brésilienne d'Anthropologie) }\end{array}$ \\
\hline 1960 & \begin{tabular}{l} 
Brasilia devient la capitale du Brésil, au lieu de Rio de Janeiro Premier Congrès brésilien de Zoologie \\
\hline
\end{tabular} \\
\hline
\end{tabular}




\section{Références bibliographiques}

Andermann, Jens (s/d). The Museu Nacional at Rio de Janeiro. Relics and Selves : Articles. Birkbeck College : $<$ http :/www.bbk.ac.uk/ibamuseum/texts/Andermann01.htm>.

Bruford, W. H., 1975. The German Tradition of Self-Cultivation. Bildung from Humboldt to Thomas Mann. Cambridge : Cambridge University Press.

CAstro, Leonardo C. de, 2003. Da Biogeografia à Biodiversidade: Políticas e Representações da Mata Atlântica. Thèse de doctorat au Programa de Pós-Graduação em Antropologia Social, Museu Nacional, Université Fédérale de Rio de Janeiro.

Corbin, Alain, 1988. Le Territoire du Vide. L'Occident et le désir du rivage (1750-1840). Paris : Aubier.

Cunha, Luiz A., 1980. A Universidade Temporã. O ensino superior da Colônia à Era Vargas. Rio de Janeiro : Editora Civilização Brasileira.

Daou, Ana Maria, 2001. « Tipos e aspectos do Brasil : imagens e imagem do Brasil ». Paisagem, Imaginário e Espaço. (Rosendahl, Zeny \& Corrêa, Roberto L.) Editora da UERJ, pp. 135-162.

Dias Duarte, Luiz F., 1999. «O Império dos Sentidos : Sensibilidade, Sensualidade e Sexualidade na Cultura Ocidental Moderna », in Maria Luiza Heilborn, (ed.) Sexualidade. O olhar das ciências sociais. Rio de Janeiro : Jorge Zahar Editor, pp. 21-30.

Dias Duarte, Luiz F., 2000. «Anthropologie, Psychanalyse et 'civilisation' du Brésil de l'entre-deux-guerres », Revue de Synthèse, 3-4 (jul.-dez.), pp. 325-344.

Dias Duarte, Luiz F.,. \& M. Jayme Aranha Filho, 2003. « Um museu de história natural na encruzilhada : a fundamentação conceitual para uma nova exposição no Museu Nacional », in José N. Bittencourt, Sarah F. Benchetrit \& Vera Lúcia B. Tostes, (eds.) História representada : o dilema dos Museus. Rio de Janeiro : Museu Histórico Nacional, pp. 197-218.

Dumont, Louis, 1983. Essais sur l'Individualisme. Une perspective anthropologique sur l'idéologie moderne. Paris : Editions du Seuil.

Dumont, Martine, 1984. «Le succès mondain d'une fausse science. La Physiognomonie de Johann Kaspar Lavater ». Actes de la Recherche en Sciences Sociales.

Elliott, Brent, 2003. Flora. An illustrated history of the garden flower. Londres: The Royal Horticultural Society, Scriptum Editions.

Freitas, Marcus Vinícius de, 2001. Hartt : Expedições pelo Brasil imperial. São Paulo : Metalivros.

Gould, Stephen J., 1987. « Deep Time and Ceaseless Motion », in An Urchin in the Storm : Essays about Books and Ideas. New York \& London : W.W. Norton \& Co, pp. 93-103.

GreEnblatt, Stephen, 1980. Renaissance Self-Fashioning. Chicago : Chicago University Press.

Gusdorf, Georges, 1974. Introduction aux Sciences Humaines. Essai critique sur leurs origines et leur développement. Paris : Ophrys.

JAMIN, Jean, 1979. « Naissance de l'observation anthropologique : la Société des Observateurs de l’Homme (17991805) ». Cahiers Internationaux de Sociologie V (67).

Jomard, E. F. et al., 1830. Description de l'Egypte. Paris : Imprimerie Royale.

Koyré, Alexandre, 1957. From the Closed World to the Infinite Universe. The Johns Hopkins Press.

Lawrence, C. J., 1979. «The nervous system and society in the Scottish Enlightenment », in Natural Order. (eds.). B. Barnes and S. Shapin : Sage Publications.

Lopes, Maria Margaret, 1997. O Brasil descobre a pesquisa cientifica - os museus e as ciências naturais no século 19. São Paulo: Hucitec.

Lovejoy, Arthur, 1993. The great chain of Being. Harvard Univ. Press. 
Mengal, Pierre, 2000. « La constitution de la psychologie comme domaine du savoir (16 $16^{\mathrm{e}} 17^{\mathrm{e}}$ siècles $)$ », Revue d'Histoire des Sciences Humaines, 2.

Nascimento, Fátima R., 1991. A Imagem do Índio na segunda metade do século 19. Thèse de Maîtrise à l'Escola de Belas Artes, Université Fédérale de Rio de Janeiro.

Nery, Paulo, 1998. Viagem, Passeio, Turismo. Estudo comparado do deslocamento como valor. Thèse de doctorat au Programa de Pós-Graduação em Antropologia Social, Museu Nacional, Université Fédérale de Rio de Janeiro.

PÁdua, José Augusto, 2002. Um sopro de destruição : pensamento político e crítica ambiental no Brasil escravista, 1786-1888. Rio de Janeiro : Jorge Zahar Ed.

Peirano, Mariza, 1981. The Anthropology of Anthropology : the case of Brazil. Universidade de Brasília.

Peirano, Mariza, 1998. «When Anthropology is at Home. The Different Contexts of a Single Discipline ». Annual Review of Anthropology, (27), pp. 105-129.

Ricotta, Lúcia, 2003. Natureza, ciência e estética em Alexander von Humboldt. Rio de Janeiro : Mauad.

SAHLINS, Marshall, 1996. "The sadness of sweetness - the native anthropology of Western Cosmology ». Current Anthropology, 37, pp. 395-415.

SAntos, Myriam S., 2000. « Os museus brasileiros e a constituição do imaginário nacional ». Brasília : Sociedade e Estado, 15 (2), pp. 271-302.

Schama, Simon, 1995. Landscape and memory. Londres : Harper \& Collins.

Schwarcz, Lília M., 1998. As Barbas do Imperador. D. Pedro II, um monarca nos trópicos. São Paulo: Companhia das Letras.

SEveri, Carlo, 1988. « Structure et Forme Originaire », in Les Idées de l'Anthropologie, org. Descola, Philippe. Paris : Armand Collin.

SEyfERth, Giralda, 1989. « As ciências sociais no Brasil e a questão racial », in Jaime Silva et al., eds. Cativeiros e liberdade. Rio de Janeiro, Editora da UERJ, pp. 11-31.

Stocking Jr., W. George, 1987. Victorian Anthropology. N.Y. : The Free Press.

Thomas, Keith, 1983. Man and the Natural World. Changing attitudes in England, 1500-1800. Harmondsworth : Penguin Books.

Viveiros de Castro, Eduardo, 1993. « Le Marbre et le Myrte : de l'Inconstance de l'Ame Sauvage », in Mémoire de la tradition. (Monod-Becquelin, Aurore, ed.). Paris : Société d'Ethnographie, 1993, pp. 365-431.

WolfF, Larry, 2003. «The Innocence and Natural Liberty of Morlacchia : European Identity, Enlightened Anthropology and the Ambivalent Significance of Gender among Noble Savages ». Dialectical Anthropology, 27, pp. 93-104. 